\title{
A Wideband Fault Location Scheme for Active Distribution Systems
}

\author{
F. M. Aboshady*†, Mark Sumner* and D. W. P. Thomas* \\ ${ }^{*}$ Department of Electrical and Electronic Engineering, University of Nottingham, Nottingham, UK \\ ${ }^{\dagger}$ Electrical Power and Machines Engineering Department, Tanta University, Tanta, Egypt \\ fathy.aboshady@nottingham.ac.uk
}

\begin{abstract}
This paper proposes a fault location technique for future distribution systems with distributed generation. The technique considers the circuit analysis at the non-fundamental frequencies that are generated when a fault occurs. To consider both inductive and capacitive parameters of the line, the distributed parameter line model is used for the analysis. A general $5^{t h}$ order polynomial is derived to estimate the fault distance and can be used for different fault types. The IEEE 34-bus feeder which is an actual distribution system is simulated and used to assess the accuracy of the method. The results obtained from different test conditions emphasize the high accuracy of the method with almost all cases having an error in estimated distance of less than $100 \mathrm{~m}$.
\end{abstract}

Index Terms-distributed generation, fault location, fault transients

\section{INTRODUCTION}

The integration of distributed generation (DG) especially renewable energy sources such as PV arrays and wind turbines has seen a rapid increase over recent years [1], [2]. From a power system point of view, this integration has economical and technical benefits such as reducing the system electrical losses and increasing the system reliability. Normally, the distribution systems are fed from a single end. However with multiple sources in the system, the power flow will no longer be uni-directional. This will have adverse effects on conventional protection systems and modifications are necessary to ensure reliability of future distribution systems [3], [4].

Fault location in distribution systems plays an important role in reducing the system outage time and therefore saving money and improving the system continuity indices [5]. Fault location techniques can be classified to impedance based, travelling wave based, artificial intelligence based and sparse measurements based techniques. The impedance based methods use the measured voltages and currents at one or more locations and analyse the circuit at the system fundamental frequency to estimate the distance to the fault point [6]-[8]. These methods are cheap to implement but suffer from multiple estimation problem when depending on measurements only at a single end [9]. The fault inception creates high frequency travelling waves that move between the fault point and the system terminals. The travelling time for these signals is used as a measure for the fault distance [10]. The travelling wave based methods require very high sampling rate and become complex with distribution systems with several laterals and loading taps. Computational intelligence techniques such as artificial neural networks (ANNs) have been used for fault location purposes [11]. A huge amount of data is required for training the ANNs and the training should be repeated when the system configuration changes. Dispersed measurements have been used to calculate the voltage sag at different points and use this information to define the fault bus [12]. Also, smart meters have been used to build a low voltage zone that contains the fault and helps in solving the multiple estimate problem of the impedance based methods [13].

For future smart distribution systems, a strong communication platform between the decentralised generation substations will be available. This paper presents a wideband fault location (WBFL) scheme that benefits from the availability of multiple measurements in future distribution systems (i.e. a double end method). The proposed WBFL scheme uses the high frequency components created by a fault to estimate the fault distance. Due to the dependence on high frequency non-fundamental frequencies, the WBFL scheme can estimate the fault distance based on a short window of data (one cycle during the fault). Therefore, it can locate fast clearing temporary faults. System disconnection is not necessary for temporary faults and the ability to locate temporary faults helps in defining the weak spots in the system to help in rescheduling maintenance. System analysis at non-fundamental frequencies has been utilized for fault location in [14]-[18]. The effect of inductive and capacitive coupling of the lines has been totally ignored in [14]-[16]. In [17], the inductive coupling has been considered however, the capacitance of the line has been ignored. In [18], the method considers both line inductive and capacitive coupling by modelling the line as $\pi$ model, however it has been developed for systems without distributed generation. In this paper, the WBFL scheme will be derived considering the distributed parameter line model which is more generic, and therefore more suitable for transmission and distribution systems.

\section{Methodology}

In this section, the proposed wideband fault location (WBFL) scheme is derived. In order to generalise the method for any line type, a distributed parameter line model has been used. In this analysis, the inductive and capacitive coupling of the line have been considered. The general representation for the distributed parameter line model is shown in Fig. 1 with 
$Z$ and $Y$ are representing the line per unit length impedance and admittance respectively.

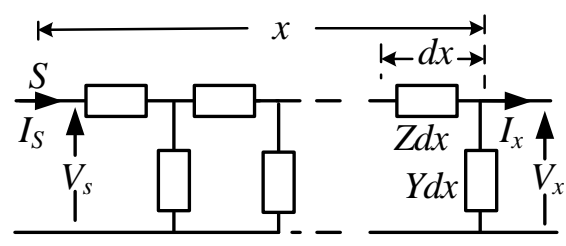

Fig. 1: Distributed parameter line

\section{A. WBFL scheme derivation}

Consider a line section between two nodes $S$ and $R$ with a fault at a distance $x$ from node $S$. The equivalent circuit at the system fundamental frequency for a fault with a resistance $R_{f}$ is shown in Fig. 2a. In the proposed method, the fault is considered as a voltage source with a step change which is equal and opposite to the voltage at the fault point at the instant of the fault. This fault voltage source $\left(V_{f}\right)$ injects non-fundamental high frequency components into the system, refer to Fig. 2b. These high frequency components are used by the proposed WBFL scheme to locate the fault distance $x$.

In Fig. 2, the voltage at the fault point $\left(V_{x}\right)$ can be calculated based on voltage and current at both ends by (1) and (2).

$$
\begin{gathered}
V_{x}=\cosh (\gamma x) V_{S}-z_{c} \sinh (\gamma x) I_{S} \\
V_{x}=\cosh (\gamma(1-x)) V_{R}+z_{c} \sinh (\gamma(1-x)) I_{R}
\end{gathered}
$$

where $\gamma$ and $z_{c}$ are the propagation constant and characteristic impedance of the line respectively and are given by (3)

$$
\gamma=\sqrt{Z Y}, z_{c}=\sqrt{Z / Y}
$$

By equating (1) and (2) and using the first three terms for both cosh and sinh expansion, a fifth order polynomial to calculate the fault distance $x$ can be obtained.

$$
\begin{aligned}
& \left(A_{S}-A_{R}\right)+\left(B_{S}-B_{R}\right) x+\left(C_{S}-C_{R}\right) x^{2} \\
& \quad+\left(D_{S}-D_{R}\right) x^{3}+\left(E_{S}-E_{R}\right) x^{4}+\left(F_{S}-F_{R}\right) x^{5}=0
\end{aligned}
$$

where values for different constants are given as follow:

$$
\begin{aligned}
& A_{S}=V_{S} \quad B_{S}=-\gamma z_{c} I_{S} \\
& C_{S}=\gamma^{2} V_{S} / 2 \quad D_{S}=-\gamma^{3} z_{c} I_{S} / 6 \\
& E_{S}=\gamma^{4} V_{S} / 24 \quad F_{S}=-\gamma^{5} z_{c} I_{S} / 120 \\
& A_{r}=V_{R} \quad B_{r}=\gamma z_{c} I_{R} \\
& C_{r}=\gamma^{2} V_{R} / 2 \quad D_{r}=\gamma^{3} z_{c} I_{R} / 6 \\
& E_{r}=\gamma^{4} V_{R} / 24 \quad F_{r}=\gamma^{5} z_{c} I_{R} / 120 \\
& A_{R}=A_{r}+B_{r}+C_{r}+D_{r}+E_{r}+F_{r} \\
& B_{R}=-\left(B_{r}+2 C_{r}+3 D_{r}+4 E_{r}+5 F_{r}\right) \\
& C_{R}=C_{r}+3 D_{r}+6 E_{r}+10 F_{r} \\
& D_{R}=-\left(D_{r}+4 E_{r}+10 F_{r}\right) \\
& E_{R}=E_{r}+5 F_{r} \quad F_{R}=-F_{r}
\end{aligned}
$$

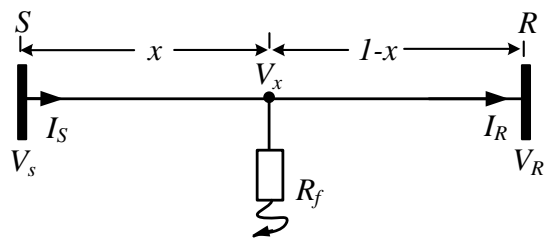

(a)

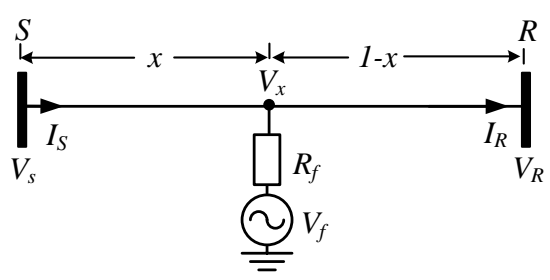

(b)

Fig. 2: Line section with fault, (a) at fundamental frequency, (b) at non-fundamental frequencies

For a three phase system, both $Z$ and $Y$ are $3 \times 3$ matrices with non zero mutual elements between phases. To apply (5), it is required to decouple the system into independent modes by using modal transformation [20]. Clarke's matrix is commonly used with symmetrical lines when converting to modal domain (6) [21], and this has been used in this paper. With asymmetrical lines, transformation matrices based on impedance and admittance eigenvectors are used. Therefore, the coefficients in (5) are calculated in the modal domain and then converted to phase domain.

$$
T_{c l}=\left[\begin{array}{ccc}
-1 / \sqrt{6} & 2 / \sqrt{6} & -1 / \sqrt{6} \\
1 / \sqrt{2} & 0 & -1 / \sqrt{2} \\
1 / \sqrt{3} & 1 / \sqrt{3} & 1 / \sqrt{3}
\end{array}\right]
$$

\section{B. Sweep of measurements}

Real distribution systems consist of many lines. In order to apply the WBFL scheme, estimates of voltage and current at both ends of the fault section are required (nodes $S$ and $R$ in Fig. 2). This can be accomplished by sweeping the measured voltage and current at both feeder ends to the assumed fault section terminals. To sweep downstream from the the start node of the feeder, (7) and (8) can be used, refer to Fig. 3a. On the other hand, (9) and (10) can be used for the upstream sweep, refer to Fig. $3 \mathrm{~b}$.

$$
\begin{gathered}
{\left[\begin{array}{c}
V_{k+1} \\
I_{k+1}^{\prime}
\end{array}\right]=\left[\begin{array}{cc}
\cosh (\gamma l) & -z_{c} \sinh (\gamma l) \\
-\sinh (\gamma l) / z_{c} & \cosh (\gamma l)
\end{array}\right]\left[\begin{array}{l}
V_{k} \\
I_{k}
\end{array}\right]} \\
I_{k+1}=I_{k+1}^{\prime}-Y_{L(k+1)} V_{k+1}+I_{d g(k+1)} \\
{\left[\begin{array}{c}
V_{k} \\
I_{k}
\end{array}\right]=\left[\begin{array}{cc}
\cosh (\gamma l) & z_{c} \sinh (\gamma l) \\
\sinh (\gamma l) / z_{c} & \cosh (\gamma l)
\end{array}\right]\left[\begin{array}{c}
V_{k+1} \\
I_{k+1}^{\prime}
\end{array}\right]} \\
I_{k}^{\prime}=I_{k}+Y_{L(k)} V_{k}-I_{d g(k)}
\end{gathered}
$$

where $l$ is the length of the section between nodes $k$ and $k+1$ and $Y_{L(j)}$ is the load or equivalent admittance at node $j$. If no 
DG is connected at node $k$ or $k+1$, then the DG current in the corresponding equation is set to zero, otherwise a measurement of the DG current is required.

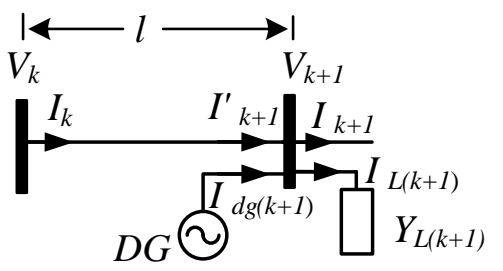

(a)

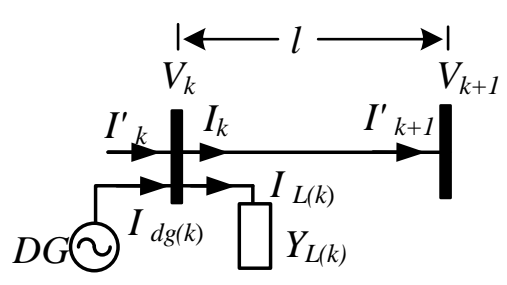

(b)

Fig. 3: Sweep of measurements, (a) downstream, (b) upstream

\section{Data processing and fault location procedure}

The voltage and current signals at the main substation and at different DG locations are measured with a sampling frequency of $20 \mathrm{kHz}$ for a period of $40 \mathrm{~ms}$ composed of 20 $\mathrm{ms}$ pre-fault and $20 \mathrm{~ms}$ during the fault. Then, the signals are processed using the Fast Fourier Transform to calculate the high frequency components required for the fault distance estimation. The distance $x$ can be calculated by (4) at each frequency considered in the frequency range (up to $3 \mathrm{kHz}$ ) and the final fault distance will be the average over the frequency range of interest. Five roots are obtained from (4), the correct root should be real positive and less than the total section length.

For a three phase system, applying (4) produces a value for each phase. The value(s) that correspond to the fault phase(s) are to be used. In case of fault combining two or three phases, the final distance will be the average.

The WBFL scheme always assumes the fault lies in the first section of the main feeder. If the estimated distance exceeds the total length of the assumed fault section, the WBFL scheme is applied again on the adjacent section until the fault is located inside the considered section. This double end method provides only one candidate location for the fault point [17]. For faults along a lateral, applying this double end method on the main feeder provides the possible fault location close to the lateral tapping point [17]. In this case, based on the availability of measurements on the lateral, either the proposed double end or a single end method [17] could be applied to locate the fault along the lateral and this procedure is illustrated in Fig. 4.

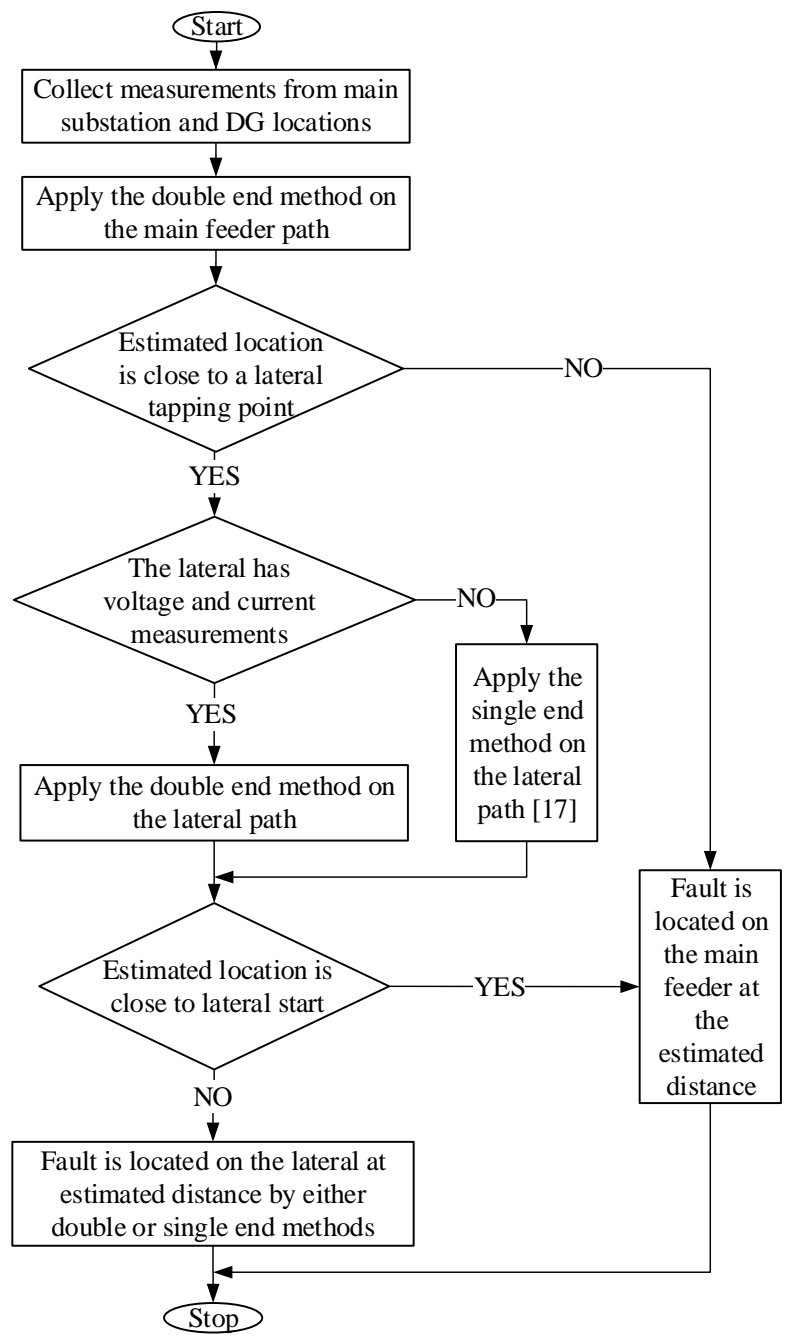

Fig. 4: Procedure of the WBFL scheme

\section{Simulation STUdies}

The proposed WBFL scheme has been implemented on the IEEE 34-bus feeder [19], refer to Fig. 5. This feeder is a real distribution feeder and exhibits unbalanced loads, non-homogeneous feeder sections and single and three-phase laterals. In this study, the main feeder is assumed to extend between the main substation (node 800) and node 848 with a total main feeder length of $58 \mathrm{~km}$. For the different test cases, the error in the estimated fault distance is calculated by (11).

$$
\text { error }=\mid \text { actual distance }- \text { estimated distance } \mid
$$

\section{A. Effect of fault distance}

The system has been modified by connecting two inverter based distributed generations (IBDGs) at nodes 828 and 848 . The IBDG has been connected to the IEEE system through LCL filter and a step up transformer. The parameters for each DG are provided in Table I. The DC side of the inverter was connected to a DC voltage source. Single line to ground (SLG), phase to phase and three-phase faults with fault resistance values of 10 and $100 \Omega$ and fault inception angle 


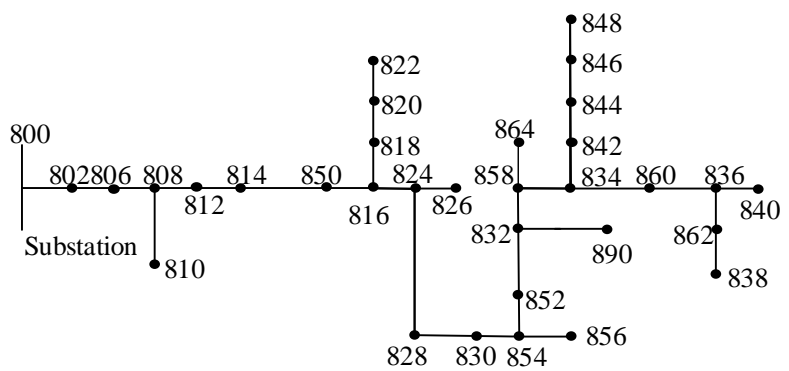

Fig. 5: Test feeder, IEEE 34-bus

of $45^{\circ}$ have been simulated at different points along the main feeder. The error in the estimated distance is shown in Fig. 6. With a maximum error of $102 \mathrm{~m}$ obtained at these conditions, the results show a robust performance for the WBFL scheme against fault distance.

TABLE I: Parameters of the IBDG

\begin{tabular}{c|c}
\hline rated power & $250 \mathrm{~kW}$ \\
\hline DC link voltage & $480 \mathrm{~V}$ \\
\hline rated line to line AC voltage & $250 \mathrm{~V}$ \\
\hline switching frequency & $3 \mathrm{kHz}$ \\
\hline inverter side inductance & $0.16 \mathrm{mH}$ \\
\hline grid side inductance & $0.04 \mathrm{mH}$ \\
\hline filter capacitance & $212 \mu \mathrm{F}$ \\
\hline damping resistance & $0.23 \Omega$ \\
\hline
\end{tabular}

\section{B. Effect of fault resistance}

In addition to the previously connected IBDGs at nodes 828 and 848 , another synchronous based DG has been connected at node 840 . A standard $250 \mathrm{kVA}$ synchronous machine model from Matlab tools has been used. The three fault types have been simulated with 25 random fault resistance values at three locations in the system. These locations lie at distance of 0.7 , 27 and $55 \mathrm{~km}$ from the main substation and will be referred to as Loc1, Loc2 and Loc3. The fault inception angle was maintained constant as $45^{\circ}$. The results for different cases are illustrated in Fig. 7 with a maximum error of $86 \mathrm{~m}$ and total average error of $20 \mathrm{~m}$.

\section{Effect of fault inception angle}

The fault inception angle is the voltage angle at the instant of the fault. To examine the effect of the fault inception angle on the performance of the proposed WBFL scheme, 25 random values between $0^{\circ}$ and $180^{\circ}$ have been used. With a fault resistance of $10 \Omega$, the three fault types have been simulated at Loc1, Loc2 and Loc3 and the results are presented in Fig. 8. As is clear, all cases produce an error of less than $100 \mathrm{~m}$ (maximum is $94 \mathrm{~m}$ ) and the total average error is $29 \mathrm{~m}$.

\section{Fault on lateral}

As an example to illustrate the concept of using the proposed double end method to locate a fault on a lateral, SLG fault has been simulated at node 836 . The total fault distance is $57415 \mathrm{~m}$ and composed of $55982 \mathrm{~m}$ along the main feeder

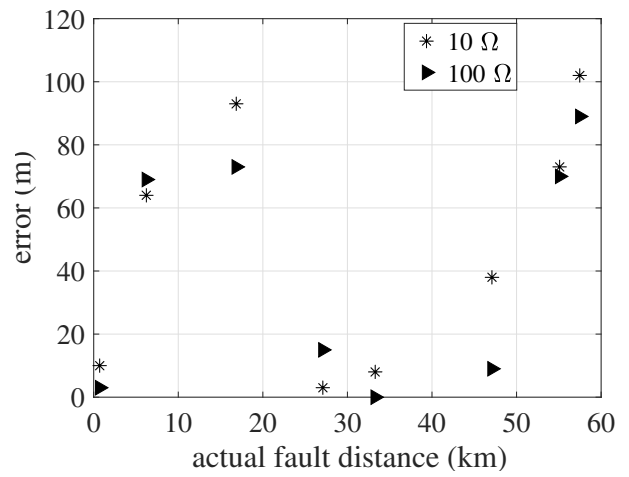

(a)

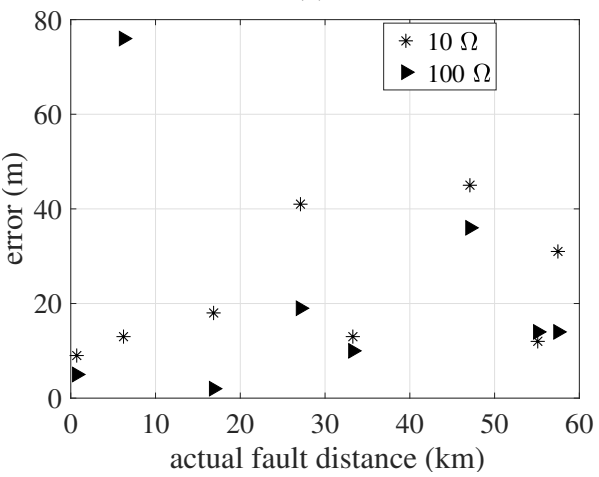

(b)

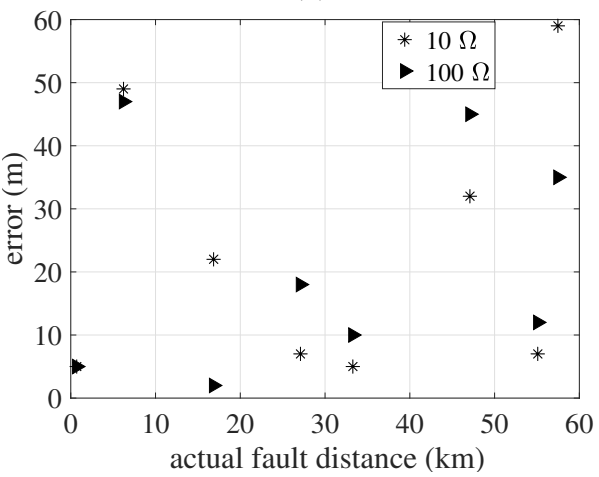

(c)

Fig. 6: Effect of fault distance: (a) SLG, (b) phase to phase, (c) three-phase faults

(from node 800 to node 834 ) and $1433 \mathrm{~m}$ along the lateral (from node 834 to node 836) as illustrated in Fig. 9.

By applying the double end method on the main feeder (from node 800 to node 848), this fault case has been located at a distance of $55789 \mathrm{~m}$ from the main substation and is indicated as $F_{1}$ in Fig. 9. The estimated fault point is not the actual fault point and the fault is located close to node 834 (the tapping point of the faulted lateral as explained earlier). In this case, as the lateral has voltage and current measurements, the double end method has been applied between the main substation (node 800) and node 840 on the lateral. The fault is located at point $F_{2}$ at a distance of $57287 \mathrm{~m}$ from the main substation which is very close to the actual fault point with an error of $128 \mathrm{~m}$ in estimation. 


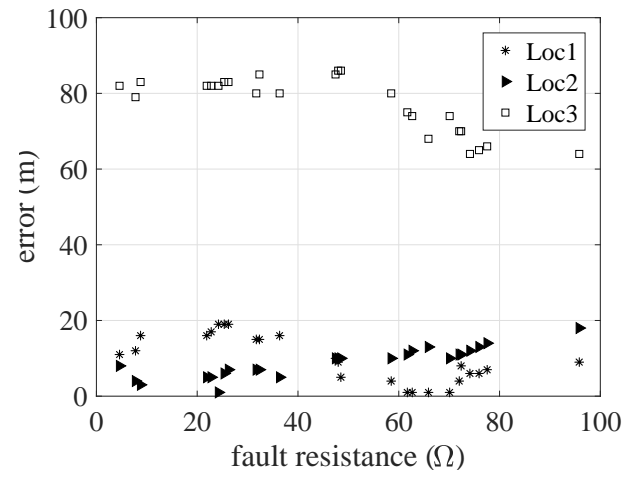

(a)

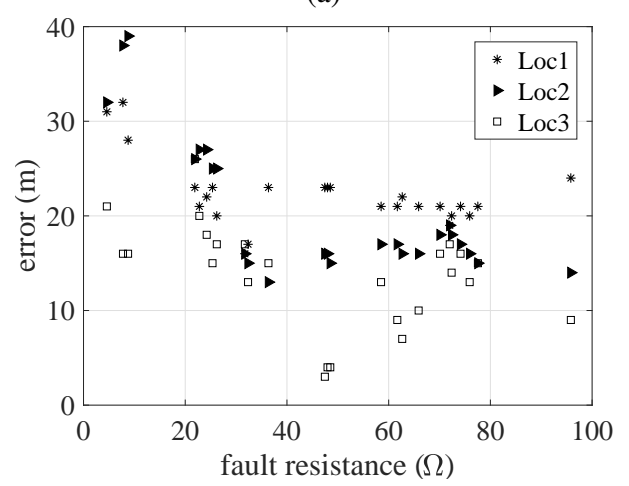

(b)

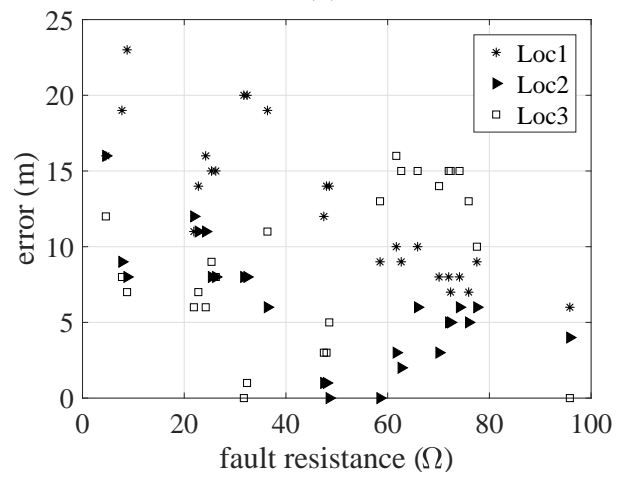

(c)

Fig. 7: Effect of fault resistance: (a) SLG, (b) phase to phase, (c) three-phase faults

All these extensive simulation studies reflects the accuracy and robustness of the proposed fault location method.

\section{CONCLUSION}

An impedance based wideband fault location scheme has been derived for distribution systems at the non-fundamental frequencies generated due to fault. The distributed parameter line model has been used in the derivation to account for both line inductive and capacitive coupling. The technique considers the presence of distributed generation and use the synchronized measurements at the main substation and different DG locations to estimate the fault distance. The derived formula is generic for any fault type and has been assessed for single line to ground, phase to phase and

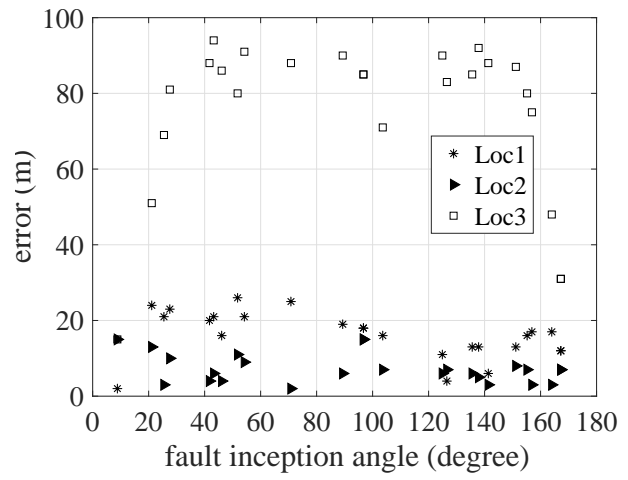

(a)

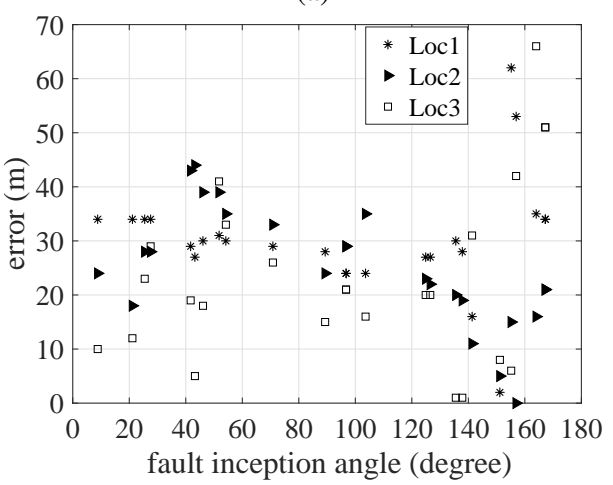

(b)

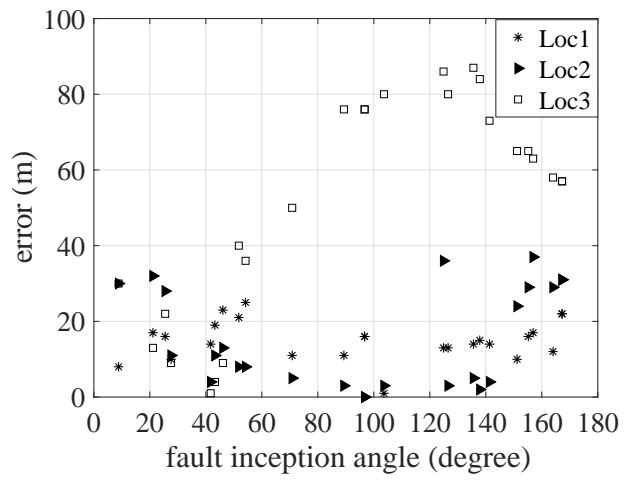

(c)

Fig. 8: Effect of fault inception angle: (a) SLG, (b) phase to phase, (c) three-phase faults

three-phase faults. In addition to locating faults along the main feeder, the availability of measurements has been employed to locate faults on the laterals which is a major problem with the traditional impedance based methods. As the analysis uses the high frequency components, a window that requires only $20 \mathrm{~ms}$ of during fault data has been used in the fault location processing allowing the proposed method to locate temporary faults as well as permanent faults. The evaluation has been carried out on a model of a real distribution feeder-IEEE 34-bus feeder. The assessment considered changing the fault point, type, resistance and inception angle. Under different test scenarios, the proposed method can estimate the fault distance with error of less than $100 \mathrm{~m}$ for almost all cases and a total 


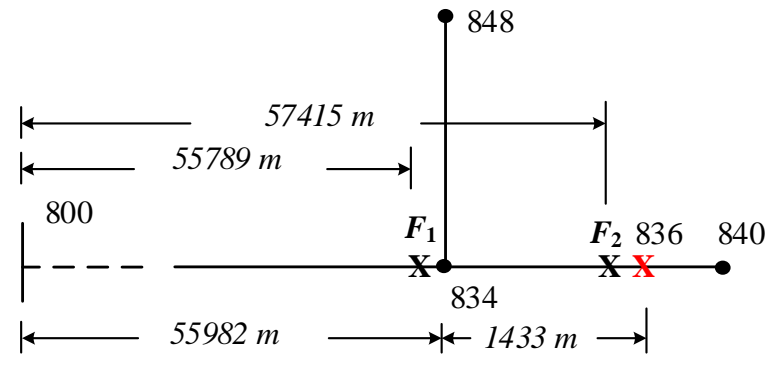

Fig. 9: Locating fault along a lateral

absolute average error of $25 \mathrm{~m}$. Also, $85 \%$ of cases were located with error in estimation of less than $50 \mathrm{~m}$ which is very low for a feeder with $93 \mathrm{~km}$ total line lengths and 58 $\mathrm{km}$ main feeder length and that reflects the accuracy of the proposed method.

\section{ACKNOWLEDGMENT}

This work has been supported by the Egyptian government (Ministry of Higher Education) and the British Council through Newton-Mosharafa PhD scholarships.

\section{REFERENCES}

[1] A. Z. Aktas, "A Review and comparison of renewable energy strategies or policies of some countries," International Conference on Renewable Energy Research and Applications (ICRERA), pp. 636-643, 2015.

[2] S. Essallah, A. Bouallegue and A. Khedher, "Optimal sizing and placement of DG units in radial distribution system," International Journal of Renewable Energy Research, vol. 8, no. 1, 2018.

[3] Y. N. Lucky de Marco, T. Zheng and S. Nikolovski, "Overcurrent protection assessment with high PV penetration in a distribution network," International Journal of Renewable Energy Research, vol. 8, no. $1,2018$.

[4] K. A. Wheeler, M. Elsamahy, and S. O. Faried, "A Novel reclosing scheme for mitigation of distributed generation effects on overcurrent protection," IEEE Transactions on Power Delivery, vol. 33, pp. 981-991, 2018.

[5] M. M. Saha, J. Izykowski, and E. Rosolowski, Fault location on power networks: Springer, 2009.

[6] F. M. Abo-Shady, M. A. Alaam, and A. M. Azmy, "Impedance-based fault location technique for distribution systems in presence of distributed generation," IEEE International Conference on Smart Energy Grid Engineering (SEGE), pp. 1-6, 2013.

[7] J. J. Mora-Florez, R. A. Herrera-Orozco, and A. F. Bedoya-Cadena, "Fault location considering load uncertainty and distributed generation in power distribution systems," IET Generation, Transmission \& Distribution, vol. 9, pp. 287-295, 2015.

[8] C. Orozco-Henao, A. S. Bretas, R. Chouhy-Leborgne, A. R. Herrera-Orozco, and J. Marín-Quintero, "Active distribution network fault location methodology: A minimum fault reactance and Fibonacci search approach," International Journal of Electrical Power \& Energy Systems, vol. 84, pp. 232-241, 2017.

[9] R. Dashti and J. Sadeh, "Fault section estimation in power distribution network using impedance-based fault distance calculation and frequency spectrum analysis," IET Generation, Transmission \& Distribution, vol. 8, pp. 1406-1417, 2014.

[10] A. Dwivedi and X. Yu, "Fault location in radial distribution lines using travelling waves and network theory," IEEE International Symposium on Industrial Electronics, pp. 1051-1056, 2011.

[11] J. J. Mora, G. Carrillo, and L. Perez, "Fault location in power distribution systems using ANFIS Nets and current patterns," IEEE/PES Transmission \& Distribution Conference and Exposition: Latin America, pp. 1-6, 2006.
[12] S. Lotfifard, M. Kezunovic, and M. J. Mousavi, "Voltage sag data utilization for distribution fault location," IEEE Transactions on Power Delivery, vol. 26, pp. 1239-1246, 2011.

[13] F. C. L. Trindade and W. Freitas, "Low voltage zones to support fault location in distribution systems with smart meters," IEEE Transactions on Smart Grid, vol. 8, pp. 2765-2774, 2017.

[14] K. Jia, D. Thomas, and M. Sumner, "A New single-ended fault-location scheme for utilization in an integrated power system," IEEE Transactions on Power Delivery, vol. 28, pp. 38-46, 2013.

[15] K. Jia, D. W. P. Thomas, and M. Sumner, "A New double-ended fault-location scheme for utilization in integrated power systems," IEEE Transactions on Power Delivery, vol. 28, pp. 594-603, 2013.

[16] K. Jia, T. Bi, Z. Ren, D. Thomas, and M. Sumner, "High frequency impedance based fault location in distribution system with DGs," IEEE Transactions on Smart Grid, vol. 9, pp. 807-816, 2016.

[17] F. M. Aboshady, D. W. P. Thomas, and M. Sumner, "Fast fault location scheme for distribution systems based on fault transients," IET International Conference on Resilience of Transmission and Distribution Networks (RTDN 2017), pp. 1-6, 2017.

[18] F. M. Aboshady, M. Sumner, and D. W. P. Thomas, "A double end fault location technique for distribution systems based on fault-generated transients," IEEE 26th International Symposium on Industrial Electronics (ISIE), pp. 32-36, 2017.

[19] IEEE PES AMPS DSAS Test Feeder Working Group, 34-bus feeder[Online] Available: http://sites.ieee.org/pes-testfeeders/resources.

[20] L. Wedepohl, "Application of matrix methods to the solution of travelling-wave phenomena in polyphase systems," in Proceedings of the Institution of Electrical Engineers, IET, vol. 110, pp. 2200-2212, 1963.

[21] A. Prado, S. Kurokawa, J. Pissolato Filho, and L. Bovolato, "Step by step analyses of Clarke's matrix correction procedure for untransposed three-phase transmission line cases," in Power and Energy Society General Meeting, IEEE, pp. 1-9, 2010. 